\title{
Genetic Variability for Seed Yield, Oil Content and Fatty Acid Composition in Germplasm Accessions of Sunflower (Helianthus annuus L.) and their Response to Different Seasons
}

\author{
H. G. Praveen*, T. K. Nagarathna, M. Gayithri and Manjugouda I Patil \\ Department of Crop Physiology, University of Agricultural Sciences, \\ GKVK, Banglore-560065, India \\ *Corresponding author
}

\begin{tabular}{|c|c|}
\hline & A B S T R A C T \\
\hline & \multirow{6}{*}{$\begin{array}{l}\text { An investigation was carried out to study the genetic variability and effect of } \\
\text { environmental factors on oil content and fatty acid composition in two different seasons } \\
\text { using } 33 \text { sunflower accessions. Significant variability for physiological, morphological, } \\
\text { yield and yield attributing characters and also for oil content and fatty acid composition } \\
\text { due to the effect of seasons, temperature and genetic makeup of genotypes were observed. } \\
\text { Plants grown in kharif had } 36.4 \text { per cent more grain yield compared to rabi. Hybrids } \\
\text { yielded more followed by inbreds and seed setting was less in RHA lines. Higher TDM } \\
\text { was recorded with hybrids compared to CMS lines, RHA-lines and inbreds. Out of } 33,22 \\
\text { genotypes recorded higher oil percentage in rabi than in kharif. Oil content was less in } \\
\text { most of the genotypes during rabi/summer due to physiological maturity of genotypes } \\
\text { coincided with higher temperature and bright sunshine hours. Accumulation of maximum } \\
\text { grain oil content was found in early matured genotypes. Depending on their consistent } \\
\text { performance, genotypes can be selected for heterosis breeding to develop good fatty acid } \\
\text { containing hybrids combined with high seed yield. }\end{array}$} \\
\hline & \\
\hline $\begin{array}{l}\text { Sunflower, Fatty } \\
\text { acid composition, } \\
\text { Gx E interaction, } \\
\text { Oil content }\end{array}$ & \\
\hline Article I & \\
\hline $\begin{array}{l}\text { Accepted: } \\
\text { 20 May } 2018 \\
\text { Available Onli } \\
\text { 10 June } 2018\end{array}$ & \\
\hline & \\
\hline
\end{tabular}

\section{Introduction}

Sunflower is a rich source of edible oil (28$45 \%$ ). Oil stability, plasticity and nutritional quality are mainly determined by fatty acid composition. In recent years, the increasing demand for vegetable oils has renewed interest in quality of sunflower oil. The areas of sunflower production vary for climate, weather (rainfall and temperature) and other environmental factors such as intercepted solar radiation, altitude, and latitude and soil type. Fatty acid composition of sunflower seeds is determined by plant genotype and more or less affected by environmental conditions such as light and temperature (Schulte et al., 2013).

Temperature and the amount of moisture in the soil are the major factors influencing sunflower seed oil composition and especially oleic acid content (Baldini et al., 2002). Fatty acid composition of sunflower is also affected by the genotype and its interaction with the environment. Genotype by environment interaction (GXE) has also been reported (Lajara et al., 1990). As a result, the study of $\mathrm{G} \times \mathrm{E}$ interaction for sunflower hybrids is 
necessary in order to select stable and widely adapted hybrids in production areas. Among several environmental factors, temperature is the main environmental factor affecting not only plant growth, development and productivity but also fatty acid composition in sunflower (Piva et al., 2000). The change in the temperature especially from anthesis to maturity has a profound effect on oleic to linoleic acid ratio in the seeds. This is due to the response of oleate desaturase enzyme to the temperature causing change in the accumulation of oleic and linoleic acids (Flagella et al., 2002, Grompone, 2005). However, the duration of this temperature effect varies with plant species, while in sunflower seeds the percentage of linoleic acid is highly influenced by growth temperature (Lajara et al., 1990).

Due to large influence of genotype and environmental conditions on oil content and fatty acid composition, it is necessary to analyze the stability of genotypes also which should be stable irrespective of the growing environment. In this regard, an investigation was carried out to assess the performance of different sunflower accessions for oil content and fatty acid composition to a change in weather and seasons.

\section{Materials and Methods}

A field experiment was conducted during kharif 2013 and rabi/summer 2013-14 at All India Coordinated Research Project on Sunflower, ZARS, UAS, GKVK, Bengaluru with 33 sunflower germplasm accessions comprising hybrids (3), CMS-lines (8), RHAlines (7) and inbreds (5) (Table 1). These accessions were selected from our previous research based on the accumulation of oleic and linoleic acids using simple sequence repeat markers (SSR markers) with confirmation using gas chromatography (Nagarathna et al., 2011).
During the experimental period in kharif the Tmax was $27.7^{\circ} \mathrm{C}$ and $\mathrm{Tmin} 19^{\circ} \mathrm{C}$ with an average rainfall about $503 \mathrm{~mm}$. Similarly in rabi $28^{\circ} \mathrm{C}$ was recorded as maximum temperature and $15.5^{\circ} \mathrm{C}$ minimum temperature with $10 \mathrm{~mm}$ rainfall.

During the crop growth period, several physiological and morphological observations were recorded. Days to 50 per cent flowering are an important trait to synchronize the flowering time and to know the duration of the crop after flowering. The number of days required for the florets to appear in 50 per cent of the plants in each plot was recorded as days to 50 per cent flowering. Total leaf area, an indication of total photosynthesizing area of the plant was measured following nondestructive method proposed for sunflower by Nanja Reddy et al., (1995).

To get seed yield per plant, seeds were separated from the head, dried and weighed and expressed as gram per plant. Completely dried hundred seeds randomly sampled from all the harvested plants from each genotype were counted and weighed to 100 seed weight.

Using $100 \mathrm{ml}$ volumetric flask hundred $\mathrm{ml}$ of well-filled seeds obtained after threshing and cleaning was recorded on dry weight basis, to obtain seed weight on volume basis (cc per100 $\mathrm{ml})$.

To record total dry weight, completely dried leaves, stem and thalamus of individual plants from each genotype was recorded separately using weighing balance.

Harvest index was determined using the following formula and expressed the values in percentage.

Harvest index $=\frac{\text { Economical Yield }}{\text { Biological yield }}$


Approximately $30 \mathrm{~g}$ of properly dried random seeds from each genotype was taken to estimate oil content using pulsed Nuclear Magnetic Resonance (NMR) spectrometer, available at AICRP Sunflower, UAS, Bengaluru which gives direct values of oil content in the seeds in terms of percentage.

All the germplasm lines were analyzed for oleic acid, linoleic acid, palmitic acid and stearic acid using gas chromatography (Agilent technologies 7890A) fitted with DBwax column (30 m long and $0.25 \mu \mathrm{m}$ ) packed with polymer of methyl silicon, using flame ionization detector (FID). The conditions maintained were column temperature: $150^{\circ} \mathrm{C}$ $270^{\circ} \mathrm{C}$, injector temperature: $250^{\circ} \mathrm{C}$ and detector temperature, $250^{\circ} \mathrm{C}$. GLC was programmed for the temperature at the rate of $10^{\circ} \mathrm{C}$ per minute increase and finally it was maintained at $270^{\circ} \mathrm{C}$.

Data were analyzed statistically using analysis of variance according to Gomez and Gomez (1984) procedure for a randomized complete block design.

\section{Results and Discussion}

\section{Days to 50 per cent flowering}

Days to 50 per cent flowering varied from as low as 53 days to 66 days. On an average more number of days was taken by hybrids, CMS lines and inbreds compared to RHA lines (Table 2). Hence, the RHA lines were short duration genotypes. Though the results were non-significant in a few entries, reduced number of days to 50 percent flowering during rabi/summer 2014 was due to higher temperature at reproductive stage which stimulated the induction of reproductive phase early. Days to flowering in most of the accessions were found to be longer in kharif. However days to flowering in some genotypes viz., GMU-702, GKVK-2, GPR-17, GPR-47,
GPR-55, RHA-23 were found to be longer in rabi/summer than in kharif due to interaction between genotypes and the season which might have affected the days to flowering.

\section{Total leaf area}

More total leaf area per plant was recorded in kharif compared to rabi/summer by 20 per cent. Among all the genotypes, hybrids produced broader leaves and hence the maximum leaf area. RHA lines showed lesser leaf area per plant due to less number of leaves.

The mean between two seasons revealed that, maximum values were recorded in hybrids (12654.6) followed by other accessions $(10,000)$. Lesser leaf area $\left(\mathrm{cm}^{2} /\right.$ plant $)$ was recorded in indreds (2590) followed by RHA lines (2629.5) and more reduction in rabi/summer was observed in hybrids (30.7 $\%$ ) followed by inbreds (28\%).

\section{Seed Yield}

The data on seed yield per plant (Table 3) indicates that maximum mean yield was recorded in kharif (32.6g) compared to rabi/summer (20.7g). Hybrids which remained for longer duration in the field produced more yield than other genotypes. Hybrids produced more seed yield with a mean 50 in kharif and 35 in rabi/summer followed by inbreds and CMS lines. RHA lines seem to be low yielders (27g in kharif and $17 \mathrm{~g}$ in rabi/summer) compared to other genotypes. Among hybrids KBSH-1 yielded more with $53.4 \mathrm{~g}$ in kharif and $32 \mathrm{~g}$ in rabi/summer followed by RSFH-1 which was on par with KBSH-44. Among CMS lines, CMS-103A showed maximum seed yield which was on par with hybrids followed by CMS-54A. In addition, ID3/147/3-163 also recorded maximum seed yield $(48.7 \mathrm{~g}$ in kharif and $29.6 \mathrm{~g}$ in rabi/summer 2013-14). Though the mean seed 
yield value was less in RHA lines some of them viz. RHA-341 and RHA-308 recorded maximum seed yield per plant. The more favorable rainfall in kharif resulted in higher number of seeds per head, 100 seed weight and seed volume increased the seed yield.

\section{Test weight}

The data on 100 achene weight (test weight) presented in Table 3 shows non-significant differences between seasons. In kharif, the mean was marginally higher (4.53) than $\mathrm{rabi} / \mathrm{summer}$ (4.4) and also with the range. However, around 8 per cent reduction was recorded in hybrids in rabi/summer and it was 12 per cent in CMS lines.

Inbreds were least affected by seasons. In KBSH-1, CMS-335A, GKVK-2, GPR-29 and RHA-88, 100 achene weight was increased in rabi/summer whereas, in most of the accessions it was higher in kharif. In KBSH-1, though 100 achene weights was less in kharif by 14 per cent, seed yield maintained to be higher compared to rabi/summer 2013-14 due to higher seed number per head. RHA lines showed very less test weight compared to all other genotypes (around $3 \mathrm{~g}$ ) and least was recorded in RHA-87 (1.9).

\section{Total dry matter}

Maximum mean total dry matter was recorded in kharif (91g) which was 16 per cent more than rabi/summer crop. Hybrids showed 23.6 per cent more TDM in kharif followed by CMS lines and RHA lines (around 15 per cent) and least TDM was recorded in inbreds and the percent reduction in rabi/summer was less (13 per cent) compared to kharif. Maximum TDM was observed in KBSH-44 due to its vigorous growth followed by CMS17A (170.6g) and CMS-335A (140.4g) among CMS lines.

The mean value among RHA lines was less due to their reduced plant height, lesser number of leaves with less total plant leaf area. The least TDM was recorded in GKVK2 in both the seasons (Table 4). The values for TDM ranged from 32.8 to 217 in kharif with a mean 91 and $27.1 \mathrm{~g}$ to 170 with a mean 76 in $\mathrm{rabi} / \mathrm{summer}$ 2013-14. The average values from two seasons shows that in RHA lines the least TDM (68) followed by inbreds (80.7).

However, these plants maintained same trend in both the seasons indicating TDM was not affected by seasons whereas; more reduction (24 percent) was recorded in hybrids.

Table.1 Classification of 33 Sunflower genotypes as hybrids, CMS-lines, RHA lines and inbreds

\begin{tabular}{|c|c|c|c|c|c|}
\hline SI No. & Hybrids & & RHA-lines & & \\
\hline $\mathbf{1}$ & KBSH-41 & 1 & 95-C-1 & 14 & RHA-87 \\
\hline $\mathbf{2}$ & KBSH-44 & 2 & GKVK-2 & 15 & RHA-88 \\
\hline $\mathbf{3}$ & RSFH-1 & 3 & GPR-11 & 16 & RHA-341 \\
\hline & CMS-lines & 4 & GPR-14 & 17 & RHA-308 \\
\hline $\mathbf{1}$ & CMS-57A & 5 & GPR-17 & & Inbreds \\
\hline $\mathbf{2}$ & CMS-54A & 6 & GPR-24 & 1 & GMU-702 \\
\hline $\mathbf{3}$ & CMS-17A & 7 & GPR-29 & 2 & GMU-704 \\
\hline $\mathbf{4}$ & CMS-17B & 8 & GPR-30 & 3 & DRSF 106 \\
\hline $\mathbf{5}$ & CMS-58A & 9 & GPR-47 & 4 & CSFI-5387 \\
\hline $\mathbf{6}$ & CMS-103A & 10 & GPR-55 & 5 & ID-3/147/3-163 \\
\hline $\mathbf{7}$ & CMS-135A & 11 & RCR-39 & & \\
\hline $\mathbf{8}$ & CMS-335A & 12 & RHA-23 & & \\
\hline & & 13 & RHA-89 & & \\
\hline
\end{tabular}


Table.2 Days to $50 \%$ flowering and total plant leaf area of different sunflower accessions

\begin{tabular}{|c|c|c|c|c|c|c|}
\hline \multirow{2}{*}{ Genotypes } & \multicolumn{3}{|c|}{ Days to $50 \%$ flowering } & \multicolumn{3}{|c|}{ Total leaf area $\left(\mathrm{cm}^{2 /} \mathrm{pl}\right)$} \\
\hline & Kharif & Rabi & Mean & Kharif & Rabi & Mean \\
\hline KBSH 1 & 62 & 59 & 60.5 & 6965.52 & 4351.97 & 5658.75 \\
\hline RSFH 1 & 62 & 63 & 62.5 & 8681.04 & 9891.20 & 9286.12 \\
\hline KBSH 44 & 64 & 63 & 63.5 & 15418.12 & 7286.91 & 11352.52 \\
\hline CMS $57 \mathrm{~A}$ & 62 & 59 & 60.5 & 8213.08 & 10627.47 & 9420.28 \\
\hline CMS 54 A & 66 & 65 & 65.5 & 4942.60 & 4392.06 & 4667.33 \\
\hline CMS $17 \mathrm{~A}$ & 61 & 60 & 60.5 & 7190.96 & 6049.58 & 6620.27 \\
\hline CMS 17 B & 66 & 65 & 65.5 & 5818.73 & 4369.91 & 5094.32 \\
\hline CMS 58A & 65 & 63 & 64 & 4830.24 & 2718.88 & 3774.56 \\
\hline CMS 103A & 64 & 60 & 62 & 7383.13 & 4867.47 & 6125.30 \\
\hline CMS 135A & 60 & 60 & 60 & 8790.01 & 5010.62 & 6900.32 \\
\hline CMS 335A & 61 & 62 & 61.5 & 10701.09 & 6657.03 & 8679.06 \\
\hline GMU 702 & 54 & 56 & 55 & 5571.32 & 3852.11 & 4711.72 \\
\hline GMU 704 & 65 & 65 & 65 & 6121.48 & 3001.19 & 4561.34 \\
\hline DRSF 106 & 62 & 60 & 61 & 13246.14 & 7907.26 & 10576.70 \\
\hline CSFI 5387 & 54 & 56 & 55 & 7898.42 & 7019.47 & 7458.95 \\
\hline IB/3/147 & 60 & 56 & 58 & 6011.70 & 2607.92 & 4309.81 \\
\hline 95-C-1 & 55 & 55 & 55 & 6033.15 & 6770.42 & 6401.79 \\
\hline GKVK 2 & 54 & 57 & 55.5 & 2585.40 & 2586.52 & 2585.96 \\
\hline GPR 11 & 62 & 61 & 61.5 & 6792.95 & 5966.78 & 6379.87 \\
\hline GPR 14 & 63 & 64 & 63.5 & 3576.89 & 2678.03 & 3127.46 \\
\hline GPR 17 & 51 & 57 & 54 & 4620.90 & 3766.38 & 4193.64 \\
\hline GPR 24 & 63 & 61 & 62 & 5112.79 & 4130.58 & 4621.69 \\
\hline GPR 29 & 56 & 55 & 55.5 & 6948.84 & 2211.04 & 4579.94 \\
\hline GPR 30 & 58 & 57 & 57.5 & 6252.56 & 9166.03 & 7709.30 \\
\hline GPR 47 & 56 & 59 & 57.5 & 4783.70 & 3374.03 & 4078.87 \\
\hline GPR 55 & 55 & 60 & 57.5 & 10235.37 & 8854.22 & 9544.80 \\
\hline RCR 39 & 63 & 61 & 62 & 7475.34 & 8495.97 & 7985.66 \\
\hline RHA 23 & 55 & 59 & 57 & 6993.98 & 3049.52 & 5021.75 \\
\hline RHA 86 & 56 & 54 & 55 & 3048.06 & 6108.26 & 4578.16 \\
\hline RHA 87 & 61 & 60 & 60.5 & 3161.24 & 2949.27 & 3055.26 \\
\hline RHA 88 & 57 & 57 & 57 & 4143.54 & 2954.24 & 3548.89 \\
\hline RHA 341 & 55 & 56 & 55.5 & 10946.19 & 4572.91 & 7759.55 \\
\hline RHA 308 & 57 & 56 & 56.5 & 10282.10 & 6320.33 & 8301.22 \\
\hline Mean & 59.55 & 59.42 & 59.5 & 6993.23 & 5289.87 & 6141.55 \\
\hline Range & $51-66$ & $54-65$ & & 2585.4-15418.1 & 2211.0-10627.5 & \\
\hline CV \% & 7.79 & 8.52 & & 9.57 & 9.37 & \\
\hline SEM \pm & 9.42 & 10.27 & & 473.69 & 350.69 & \\
\hline CD@5\% & 3.27 & 3.56 & NS & 1364.56 & 1010.23 & $\mathbf{S}$ \\
\hline
\end{tabular}


Table.3 Seed yield per plant, 100 seed weight and seed volume of 33 sunflower accessions

\begin{tabular}{|c|c|c|c|c|c|c|c|c|c|}
\hline \multirow{2}{*}{ Genotypes } & \multicolumn{3}{|c|}{ Seed yield per plant (g) } & \multicolumn{3}{|c|}{100 seed weight (g) } & \multicolumn{3}{|c|}{$\begin{array}{l}\text { Seed weight on volume basis } \\
(\mathrm{g} / 100 \mathrm{ml})\end{array}$} \\
\hline & Kharif & Rabi & Mean & Kharif & Rabi & Mean & Kharif & Rabi & Mean \\
\hline KBSH 1 & 53.40 & 31.50 & 42.45 & 6.18 & 7.21 & 6.70 & 40.75 & 39.50 & 40.13 \\
\hline RSFH 1 & 49.10 & 35.80 & 42.45 & 7.00 & 5.49 & 6.25 & 38.25 & 36.00 & 37.13 \\
\hline KBSH 44 & 48.70 & 38.20 & 43.45 & 8.05 & 6.75 & 7.40 & 41.75 & 40.00 & 40.88 \\
\hline CMS 57 A & 36.80 & 19.10 & 27.95 & 5.55 & 5.46 & 5.51 & 37.75 & 36.75 & 37.25 \\
\hline CMS 54 A & 45.30 & 26.20 & 35.75 & 4.14 & 4.07 & 4.11 & 38.50 & 36.75 & 37.63 \\
\hline CMS 17 A & 34.70 & 16.80 & 25.75 & 6.09 & 5.81 & 5.95 & 43.00 & 38.50 & 40.75 \\
\hline CMS 17 B & 25.50 & 18.20 & 21.85 & 4.01 & 4.00 & 4.01 & 36.00 & 29.75 & 32.88 \\
\hline CMS 58A & 26.00 & 21.00 & 23.50 & 4.62 & 4.08 & 4.35 & 38.25 & 39.00 & 38.63 \\
\hline CMS 103A & 48.60 & 35.70 & 42.15 & 6.29 & 5.85 & 6.07 & 40.75 & 40.50 & 40.63 \\
\hline CMS 135A & 36.80 & 17.60 & 27.20 & 6.10 & 4.96 & 5.53 & 39.75 & 34.25 & 37.00 \\
\hline CMS 335A & 26.60 & 17.20 & 21.90 & 7.33 & 3.03 & 5.18 & 39.25 & 28.50 & 33.88 \\
\hline GMU 702 & 31.00 & 17.60 & 24.30 & 7.91 & 7.45 & 7.68 & 48.00 & 44.00 & 46.00 \\
\hline GMU 704 & 31.50 & 24.20 & 27.85 & 2.23 & 3.22 & 2.73 & 25.25 & 26.00 & 25.63 \\
\hline DRSF 106 & 29.00 & 27.30 & 28.15 & 7.33 & 7.95 & 7.64 & 39.25 & 35.75 & 37.50 \\
\hline CSFI 5387 & 46.60 & 22.00 & 34.30 & 5.77 & 4.41 & 5.09 & 47.25 & 30.40 & 38.83 \\
\hline IB/3/147 & 48.70 & 29.60 & 39.15 & 7.53 & 6.94 & 7.24 & 32.00 & 33.00 & 32.50 \\
\hline 95-C-1 & 15.80 & 17.80 & 16.80 & 2.43 & 2.51 & 2.47 & 38.50 & 39.00 & 38.75 \\
\hline GKVK 2 & 30.30 & 25.80 & 28.05 & 2.26 & 3.94 & 3.10 & 44.50 & 44.50 & 44.50 \\
\hline GPR 11 & 17.80 & 16.80 & 17.30 & 2.84 & 2.87 & 2.86 & 44.25 & 42.50 & 43.38 \\
\hline GPR 14 & 18.10 & 13.80 & 15.95 & 3.10 & 3.02 & 3.06 & 40.75 & 42.30 & 41.53 \\
\hline GPR 17 & 20.30 & 14.40 & 17.35 & 3.37 & 3.21 & 3.29 & 42.00 & 42.25 & 42.13 \\
\hline GPR 24 & 21.50 & 15.40 & 18.45 & 2.27 & 2.98 & 2.63 & 34.00 & 36.50 & 35.25 \\
\hline GPR 29 & 31.30 & 21.00 & 26.15 & 2.48 & 3.71 & 3.10 & 37.50 & 35.30 & 36.40 \\
\hline GPR 30 & 26.40 & 14.70 & 20.55 & 2.71 & 3.08 & 2.90 & 38.75 & 39.50 & 39.13 \\
\hline GPR 47 & 25.40 & 19.20 & 22.30 & 2.32 & 3.08 & 2.70 & 41.50 & 39.00 & 40.25 \\
\hline GPR 55 & 38.90 & 17.90 & 28.40 & 3.06 & 3.12 & 3.09 & 38.25 & 37.25 & 37.75 \\
\hline RCR 39 & 31.40 & 15.30 & 23.35 & 5.10 & 4.61 & 4.86 & 37.25 & 37.00 & 37.13 \\
\hline RHA 23 & 28.70 & 15.70 & 22.20 & 2.81 & 2.95 & 2.88 & 38.50 & 39.00 & 38.75 \\
\hline RHA 86 & 17.50 & 16.00 & 16.75 & 2.81 & 2.52 & 2.67 & 40.25 & 41.50 & 40.88 \\
\hline RHA 87 & 16.00 & 14.30 & 15.15 & 1.91 & 1.90 & 1.91 & 41.75 & 40.75 & 41.25 \\
\hline RHA 88 & 22.80 & 15.70 & 19.25 & 2.13 & 4.96 & 3.55 & 43.50 & 40.00 & 41.75 \\
\hline RHA 341 & 44.40 & 16.30 & 30.35 & 5.67 & 5.39 & 5.53 & 36.00 & 39.00 & 37.50 \\
\hline RHA 308 & 52.80 & 17.20 & 35.00 & 6.01 & 4.70 & 5.36 & 36.25 & 36.50 & 36.38 \\
\hline Mean & 32.66 & 20.77 & 26.72 & 4.53 & 4.40 & 4.47 & 39.37 & 37.59 & 38.48 \\
\hline Range & 15.8-53.4 & $13.8-38.2$ & & 1.9-8.1 & 1.9-7.9 & & $25.3-48$ & $26-44.5$ & \\
\hline CV \% & 8.17 & 8.29 & & 9.68 & 9.03 & & 7.84 & 6.61 & \\
\hline SEM & 5.44 & 3.51 & & 0.30 & 0.28 & & 2.18 & 1.75 & \\
\hline CD@5\% & 1.89 & 1.22 & $\mathbf{S}$ & 0.89 & 0.80 & NS & 6.29 & 5.06 & $\mathbf{S}$ \\
\hline
\end{tabular}


Table.4 Total dry matter, harvest index and oil content of 33 sunflower accessions

\begin{tabular}{|c|c|c|c|c|c|c|c|c|c|}
\hline \multirow{2}{*}{ Genotypes } & \multicolumn{3}{|c|}{ Total dry matter $(\mathrm{g} / \mathrm{pl})$} & \multicolumn{3}{|c|}{ Harvest index (\%) } & \multicolumn{3}{|c|}{ Oil content (\%) } \\
\hline & Kharif & Rabi & Mean & Kharif & Rabi & Mean & Kharif & Rabi & Mean \\
\hline KBSH 1 & 162.75 & 133.35 & 148.05 & 24.71 & 19.11 & 21.91 & 39.05 & 32.93 & 35.99 \\
\hline RSFH 1 & 160.10 & 108.80 & 134.45 & 23.47 & 24.76 & 24.12 & 33.89 & 35.80 & 34.85 \\
\hline KBSH 44 & 216.90 & 170.05 & 193.48 & 18.34 & 18.34 & 18.34 & 35.32 & 31.45 & 38.39 \\
\hline CMS 57 A & 142.20 & 105.90 & 124.05 & 20.56 & 15.28 & 17.92 & 40.93 & 36.03 & 38.48 \\
\hline CMS 54 A & 77.60 & 70.85 & 74.23 & 36.86 & 27.00 & 31.93 & 35.70 & 36.32 & 36.01 \\
\hline CMS $17 \mathrm{~A}$ & 170.63 & 128.45 & 149.54 & 16.90 & 11.57 & 14.24 & 20.39 & 26.72 & 23.56 \\
\hline CMS 17 B & 119.20 & 106.40 & 112.80 & 17.62 & 14.61 & 16.12 & 17.68 & 23.67 & 20.68 \\
\hline CMS 58A & 109.20 & 94.60 & 101.90 & 19.23 & 18.17 & 18.70 & 38.35 & 40.03 & 39.19 \\
\hline CMS 103A & 90.50 & 71.50 & 81.00 & 34.94 & 33.30 & 34.12 & 35.88 & 31.15 & 33.52 \\
\hline CMS 135A & 77.08 & 71.25 & 74.17 & 32.31 & 19.81 & 26.06 & 30.59 & 32.87 & 31.73 \\
\hline CMS 335A & 140.40 & 135.95 & 138.18 & 15.93 & 11.23 & 13.58 & 28.19 & 30.79 & 29.49 \\
\hline GMU 702 & 79.45 & 77.60 & 78.53 & 28.07 & 18.49 & 23.28 & 36.31 & 36.45 & 36.38 \\
\hline GMU 704 & 74.60 & 57.15 & 65.88 & 29.69 & 29.75 & 29.72 & 27.41 & 23.29 & 25.35 \\
\hline DRSF 106 & 138.65 & 98.55 & 118.60 & 17.30 & 21.69 & 19.50 & 31.75 & 29.44 & 30.60 \\
\hline CSFI 5387 & 82.30 & 101.55 & 91.93 & 36.15 & 17.81 & 26.98 & 38.79 & 36.43 & 37.61 \\
\hline IB/3/147 & 53.25 & 37.75 & 45.50 & 47.77 & 43.95 & 45.86 & 32.14 & 30.04 & 31.09 \\
\hline 95-C-1 & 68.18 & 63.45 & 65.82 & 18.81 & 21.91 & 20.36 & 38.45 & 39.56 & 39.01 \\
\hline GKVK 2 & 32.78 & 28.25 & 30.52 & 48.03 & 47.73 & 47.88 & 41.33 & 41.51 & 41.42 \\
\hline GPR 11 & 65.70 & 62.15 & 63.93 & 21.32 & 21.28 & 21.30 & 37.36 & 33.04 & 35.20 \\
\hline GPR 14 & 39.75 & 30.65 & 35.20 & 31.29 & 31.05 & 31.17 & 40.27 & 38.20 & 39.24 \\
\hline GPR 17 & 47.95 & 31.75 & 39.85 & 29.74 & 31.20 & 30.47 & 40.88 & 41.79 & 41.34 \\
\hline GPR 24 & 69.75 & 45.08 & 57.42 & 23.56 & 25.46 & 24.51 & 30.55 & 33.57 & 32.06 \\
\hline GPR 29 & 61.10 & 37.45 & 49.28 & 33.87 & 35.93 & 34.90 & 36.36 & 39.03 & 37.70 \\
\hline GPR 30 & 50.75 & 50.80 & 50.78 & 34.22 & 22.44 & 28.33 & 40.53 & 41.70 & 41.12 \\
\hline GPR 47 & 44.80 & 32.95 & 38.88 & 36.18 & 36.82 & 36.50 & 32.23 & 34.09 & 33.16 \\
\hline GPR 55 & 64.80 & 51.40 & 58.10 & 37.51 & 25.83 & 31.67 & 29.78 & 34.23 & 32.01 \\
\hline RCR 39 & 98.60 & 82.10 & 90.35 & 24.15 & 15.71 & 19.93 & 32.22 & 34.60 & 33.41 \\
\hline RHA 23 & 71.25 & 61.50 & 66.38 & 28.71 & 20.34 & 24.53 & 30.89 & 32.18 & 31.54 \\
\hline RHA 86 & 39.45 & 28.25 & 33.85 & 30.73 & 36.16 & 33.45 & 39.49 & 41.09 & 40.29 \\
\hline RHA 87 & 34.90 & 27.15 & 31.03 & 31.43 & 34.50 & 32.97 & 38.27 & 40.36 & 39.32 \\
\hline RHA 88 & 78.30 & 63.55 & 70.93 & 22.55 & 19.81 & 21.18 & 41.07 & 29.77 & 35.42 \\
\hline RHA 341 & 129.66 & 123.90 & 126.78 & 25.51 & 11.63 & 18.57 & 33.71 & 29.07 & 31.39 \\
\hline RHA 308 & 110.25 & 118.35 & 114.30 & 32.38 & 12.69 & 22.54 & 27.84 & 28.30 & 28.07 \\
\hline Mean & 90.99 & 76.01 & 83.50 & 28.18 & 24.10 & 26.14 & 34.35 & 34.10 & 34.08 \\
\hline Range & $32.8-216.9$ & 27.1-170.1 & & $15.9-48.0$ & $11.2-47.7$ & & $17.7-41.3$ & 23.3-41.8 & \\
\hline CV \% & 11.58 & 15.14 & & 8.90 & 12.03 & & 6.30 & 4.95 & \\
\hline SEM & 7.45 & 8.14 & & 1.78 & 2.13 & & 1.52 & 1.19 & \\
\hline CD@5\% & 21.45 & 23.45 & NS & 5.11 & 6.13 & $\mathbf{S}$ & 4.38 & 3.44 & NS \\
\hline
\end{tabular}


Table.5 Fatty acid profiling of 33 sunflower accessions with their seasonal means

\begin{tabular}{|c|c|c|c|c|c|c|c|c|c|c|c|c|}
\hline \multirow{2}{*}{ Genotypes } & \multicolumn{3}{|c|}{ Oleic acid (\%) } & \multicolumn{3}{|c|}{ Linoleic acid (\%) } & \multicolumn{3}{|c|}{ Palmitic acid (\%) } & \multicolumn{3}{|c|}{ Stearic acid (\%) } \\
\hline & Kharif & Rabi & Mean & Kharif & Rabi & Mean & Kharif & Rabi & Mean & Kharif & Rabi & Mean \\
\hline KBSH 1 & 48.13 & 59.36 & 53.75 & 42.8 & 27.47 & 35.14 & 6.3 & 8.7 & 7.50 & 2.76 & 4.46 & 3.61 \\
\hline RSFH 1 & 69.52 & 79.04 & 74.28 & 21.7 & 13.79 & 17.75 & 6.03 & 5.86 & 5.95 & 2.73 & 1.29 & 2.01 \\
\hline KBSH 44 & 41.9 & 62.29 & 52.10 & 49.13 & 23.34 & 36.24 & 5.48 & 9.35 & 7.42 & 3.48 & 5.01 & 4.25 \\
\hline CMS 57 A & 40.12 & 48.64 & 44.38 & 45.05 & 43.36 & 44.21 & 9.6 & 5.6 & 7.60 & 5.21 & 2.38 & 3.80 \\
\hline CMS 54 A & 70.16 & 71.98 & 71.07 & 20.8 & 19.33 & 20.07 & 6.05 & 5.69 & 5.87 & 2.98 & 2.99 & 2.99 \\
\hline CMS 17 A & 57.38 & 58.63 & 58.01 & 29.67 & 29.07 & 29.37 & 8.96 & 8.39 & 8.68 & 3.97 & 3.89 & 3.93 \\
\hline CMS 17 B & 49.06 & 48.64 & 48.85 & 36.11 & 37.8 & 36.96 & 9.6 & 8.5 & 9.05 & 5.21 & 5.04 & 5.13 \\
\hline GMU 702 & 62.67 & 67.14 & 64.91 & 28.1 & 24.77 & 26.44 & 6.05 & 4.99 & 5.52 & 3.17 & 3.09 & 3.13 \\
\hline GMU 704 & 58.96 & 50.05 & 54.51 & 29.18 & 39.85 & 34.52 & 7.75 & 5.88 & 6.82 & 4.1 & 4.21 & 4.16 \\
\hline CMS 58A & 75.45 & 75.45 & 75.45 & 13.84 & 15.25 & 14.55 & 6.13 & 5.98 & 6.06 & 4.56 & 3.3 & 3.93 \\
\hline CMS 103A & 73.64 & 84.97 & 79.31 & 11.04 & 8.52 & 9.78 & 9.59 & 4.43 & 7.01 & 5.73 & 2.07 & 3.90 \\
\hline CMS 135A & 55.21 & 60.22 & 57.72 & 33.58 & 27.95 & 30.77 & 6.52 & 7.74 & 7.13 & 4.68 & 4.08 & 4.38 \\
\hline CMS 335A & 23.98 & 24.63 & 24.31 & 67.56 & 64.62 & 66.09 & 4.86 & 7.64 & 6.25 & 3.59 & 3.1 & 3.35 \\
\hline DRSF 106 & 41.94 & 34.05 & 38.00 & 46.61 & 51.81 & 49.21 & 8.22 & 8.86 & 8.54 & 3.22 & 5.27 & 4.25 \\
\hline CSFI 5387 & 56.51 & 32.01 & 44.26 & 32.57 & 55.59 & 44.08 & 7.38 & 8.63 & 8.01 & 3.53 & 3.76 & 3.65 \\
\hline IB/3/147 & 36.47 & 38.64 & 37.56 & 50.01 & 47.67 & 48.84 & 10.27 & 9.56 & 9.92 & 3.24 & 4.12 & 3.68 \\
\hline 95-C-1 & 50.19 & 49.19 & 49.69 & 42.04 & 43.14 & 42.59 & 7.66 & 7.56 & 7.61 & 0.09 & 0.09 & 0.09 \\
\hline GKVK 2 & 51.69 & 52.06 & 51.88 & 35.97 & 35.59 & 35.78 & 8.01 & 8.12 & 8.07 & 4.32 & 4.22 & 4.27 \\
\hline GPR 11 & 40.12 & 40.74 & 40.43 & 47.22 & 47.09 & 47.16 & 7.92 & 7.58 & 7.75 & 4.73 & 4.58 & 4.66 \\
\hline GPR 14 & 35.32 & 37.34 & 36.33 & 54.66 & 50.17 & 52.42 & 7.34 & 9.51 & 8.43 & 2.66 & 2.96 & 2.81 \\
\hline GPR 17 & 35.96 & 29.57 & 32.77 & 51.77 & 58.48 & 55.13 & 8.88 & 8.42 & 8.65 & 3.38 & 3.52 & 3.45 \\
\hline GPR 24 & 41.47 & 44.85 & 43.16 & 46.29 & 43.04 & 44.67 & 8.22 & 7.94 & 8.08 & 4.0 & 4.15 & 4.08 \\
\hline GPR 29 & 34.02 & 30.16 & 32.09 & 49.35 & 56.07 & 52.71 & 11.71 & 9.42 & 10.57 & 4.91 & 4.34 & 4.63 \\
\hline GPR 30 & 29.17 & 34.5 & 31.84 & 56.93 & 49.54 & 53.24 & 9.64 & 10.93 & 10.29 & 4.25 & 5.02 & 4.64 \\
\hline GPR 47 & 31.09 & 41.68 & 36.39 & 56.32 & 42.74 & 49.53 & 8.4 & 10.36 & 9.38 & 4.17 & 5.2 & 4.69 \\
\hline GPR 55 & 31.29 & 25.82 & 28.56 & 55.16 & 62.02 & 58.59 & 10.44 & 9.41 & 9.93 & 3.1 & 2.74 & 2.92 \\
\hline RCR 39 & 69.95 & 71.41 & 70.68 & 20.6 & 17.16 & 18.88 & 6.24 & 7.11 & 6.68 & 3.19 & 4.3 & 3.75 \\
\hline RHA 23 & 31.66 & 71.41 & 51.54 & 58.9 & 15.95 & 37.43 & 6.24 & 7.46 & 6.85 & 3.19 & 5.17 & 4.18 \\
\hline RHA 86 & 64 & 41.26 & 52.63 & 25.01 & 49.46 & 37.24 & 7.72 & 7.07 & 7.40 & 3.24 & 2.18 & 2.71 \\
\hline RHA 87 & 54.36 & 61.38 & 57.87 & 35.14 & 28.93 & 32.04 & 7.6 & 6.56 & 7.08 & 2.89 & 3.12 & 3.01 \\
\hline RHA 88 & 57.89 & 82.36 & 70.13 & 32.74 & 8.20 & 20.47 & 6.39 & 5.44 & 5.92 & 2.97 & 3.99 & 3.48 \\
\hline RHA 341 & 50.23 & 49.49 & 49.86 & 38.68 & 39.36 & 39.02 & 6.22 & 6.41 & 6.32 & 4.86 & 4.73 & 4.80 \\
\hline RHA 308 & 52.53 & 69.19 & 60.86 & 39.36 & 18.18 & 28.77 & 5.22 & 8.11 & 6.67 & 2.88 & 4.51 & 3.70 \\
\hline Mean & 49.15 & 52.37 & 50.76 & 39.51 & 36.22 & $\mathbf{3 7 . 8 7}$ & 7.66 & 7.67 & 7.67 & 3.67 & 3.72 & 3.70 \\
\hline Range & $\begin{array}{c}23.9- \\
75.5\end{array}$ & $\begin{array}{c}24.6- \\
84.9\end{array}$ & & $\begin{array}{l}11.0- \\
67.5\end{array}$ & $\begin{array}{l}8.2- \\
64.6\end{array}$ & & $4.8-1.7$ & $\begin{array}{l}4.4- \\
10.9\end{array}$ & & 0.09-5.7 & $\begin{array}{c}\text { 0.09- } \\
5.3\end{array}$ & \\
\hline
\end{tabular}

\section{Harvest index}

Considerably higher harvest index was achieved in GKVK-2 and ID-3/147/3-163 with more than 45 per cent achieved due to utilization of more photo assimilates for grain development and also they consistently performed better in both the seasons. 
Minimum HI was recorded in hybrids and a few CMS lines (CMS- 335A and CMS-17-A).

\section{Oil content}

Compared to kharif, more reduction in oil content was recorded among hybrids (15 per cent) followed by CMS lines $(10 \%)$ and the least was among RHA lines (3.9 per cent). Interestingly, early maturing genotypes recorded higher oil content and their per cent reduction between two seasons was minimal, whereas some of the hybrids and CMS lines extended number of days to 50 per cent flowering to more than 6 days. More reduction in oil content in hybrids indicates that these are more sensitive to higher temperature compared to CMS and RHA lines. KBSH-1 (39.1\%), CMS-57A (41\%) and GKVK-2 $(41.3 \%)$ produced maximum grain oil content. In some of the genotypes, (CMS135A, GPR-17, RHA 95-C-1 and RHA- 86) days to 50 per cent flowering was less (55days) and in these genotypes more oil accumulation was noticed.

The shortest duration genotypes completed their life cycle 5-11 days earlier than the other genotypes. Hence these plants did not coincide with the higher temperature at the time of harvest. In case of long duration crops, where days to 50 per cent flowering was more than 60 days were exposed to higher temperature especially from anthesis to harvest which affected the oil accumulation. During this stage the maximum temperature was 27.8 to $30^{\circ} \mathrm{C}$ whereas, it was around $27^{\circ} \mathrm{C}$ in early maturing genotypes. There was no variation in minimum temperature and did not show any major effect on the accumulation of oil content.

\section{Fatty acids}

Significant differences were observed among all the genotypes for different fatty acids. The mean value for oleic acid in hybrids is 53.2 per cent in kharif and 66.9 per cent in rabi /summer. It was 60.9 and 63.2 per cent for CMS lines and RHA lines showed 44.7 and 48.9 in kharif and rabi/summer, respectively (Table 5). The reduction in percent values between seasons was higher in hybrids. In rabi/summer 20 per cent increase in oleic accumulation was recorded in hybrids. Similarly, it was reduced to 8.6 per cent among RHA lines and the least reduction was observed in CMS lines (4.3\%). Among all the genotypes CMS-103A has maximum accumulation of oleic acid 84.9 in rabi/summer followed by RHA-88 (82.4).

Sunflower genotypes accumulated significantly different linoleic acid in two seasons. The data on linoleic acid indicate that the average linoleic accumulation was higher in kharif (39.5\%) compared to rabi/summer $(36.2 \%)$ which was inversely related to oleic content. Among all the genotypes CMS-335A accumulated higher linoleic content $(67.6 \%)$ in kharif followed by GPR-30 (56.9\%) and the least was in CMS58A (13.8 per cent). RCR-39 also showed some linoleic content with higher oleic accumulation. Among hybrids RSFH-1 has lower linoleic content and is known to be high oleic hybrid. The concentration of saturated fatty acids (palmitic and stearic acid) was not significantly influenced by seasons and temperature. The average palmitic acid and stearic acid contents were around 7.6 per cent and 3.6 per cent respectively. These two fatty acids were inversely related whereas, whenever palmitic content increased, there was a reduction in stearic content. All these results indicated that oil content and fatty acid composition, mainly oleic acid and linoleic acids are affected by climatic changes. Among all the weather factors, temperature plays a major role in the accumulation of these fatty acids. Oleic acid content was inversely related to linoleic acid. In both the seasons wherever oleic accumulated in a 
higher proportion, linoleic content reduced.

The sunflower oil contains more of unsaturated fatty acids, mainly oleic acid and linoleic acid and very less quantity of saturated fatty acids, palmitic acid and stearic. Healthy oil should contain more of unsaturated fatty acids compared to saturated fatty acids.

A significant variability was observed for all the physiological, morphological traits, yield and yield attributing characters and also for oil content and fatty acid composition in all the genotypes and also between the seasons which was due to season, temperature and genetic make up for all the physiomorphological parameters, seed yield and fatty acid accumulation. Environmental changes affect morphological, physiological, quantitative and qualitative traits in sunflower.

Temperature and bright sunshine hours during seed filling are the major determinants of oleic and linoleic acid accumulation in sunflower. The existing genetic variability for different traits can be exploited for trait specific crop improvement programme.

\section{References}

Baldini, M. R., Giovanardi, S., TahmasebiEnferadi and Vannozzi, G. P. 2002, Effects of water regimes on fatty acid accumulation and final fatty acid composition in the oil of standard and high oleic sunflower hybrids. Ital. J. Agron., 6:119-126.

Flagella Z, Rotunno T, Tarantino E, Caterina R.D and Caro A.D. 2002, Changes in seed yield and oil fatty acid composition of high oleic sunflower (Helianthus annuus L.) hybrids in relation to the sowing date and the water regime. Eur. J. Agron., 17: 221-230.

Gomez, K. A. and Gomez, A. A. 1984. Randomized complete block design analysis. In: Statistical procedures for agricultural research. John Willey and Sons, New York.

Grompone M.A. 2005, Sunflower oil. In Shahidi F (ed) Bailey's industrial oil \& fat productsedible oils and fat products: edible oils. John Wiley \& Sons, Hoboken, p. 655-730.

Lajara, J. R., Diaz and Diaz-Quidiello, U. R. 1990. Definitive influence of location and climatic conditions on fatty acid composition of sunflower seed oil. J. Amer. Oil Chemists Soc., 67: 618-623.

Nagarathna, T.K., Shadakshari, Y.G. and Ramanappa, T.M. 2011. Molecular analysis of sunflower (Helianthus annuus L.) genotypes for high oleic acid using microsatellite markers, Helia.,34, Nr. 55, p.p. 63-68.

Nanja Reddy, Y. A., Sheshshayee, M.S., Uma Shaanker, R., Virupakshappa, K. and Prasad, T. G. 1994. Selection for high canopy assimilation rate is a good strategy to increase productivity in sunflower. Helia., 17: 45-52.

Piva, Bouniols, G. A. and Mondies, G. 2000. Effect of cultural conditions on yield, oil content and fatty acid composition of sunflower kernel. Proceeding. Toulouse, France: 15th Int. Sunflower Conference. pp: 61-66.

Schulte L.R, Ballard T, Samarakoon T, Yao L, Vadlani P, Staggenborg S. and Rezac M. 2013. Increased growing temperature reduces content of polyunsaturated fatty acids in four oilseed crops. Industrial $\mathrm{Cr}$. Prod., 51: 212-219.

\section{How to cite this article:}

Praveen H. G., T. K. Nagarathna, M. Gayithri and Manjugouda I Patil. 2018. Genetic Variability for Seed Yield, Oil Content and Fatty Acid Composition in Germplasm Accessions of Sunflower (Helianthus annuus L.) and Their Response to Different Seasons. Int.J.Curr.Microbiol.App.Sci. 7(06): 2120-2129. doi: https://doi.org/10.20546/ijcmas.2018.706.252 